\title{
SULPHONE TREATMENT OF LEPROSY
}

\section{JOHN LOWE}

Sulphone therapy of leprosy has $n$ years in certain centres; almost all reports on results of treatment are favourable (Refs. I to I5).

There are one or two factors which from a theoretical standpoint might favour chemotherapy of leprosy. First, there is the 
fact that leprosy is a very chronic infection in which the vital organs of the body are practically unaffected either by the bacilli themselves directly or by toxins produced by bacilli; therefore, the chemotherapy of leprosy does not, as in other diseases, involve a race against time and an attempt to get the infection under control within a limited period before irreparable damage is done. Secondly, there are strong indications of considerable immunity to leprosy in human beings; for example, the difficulty with which the disease is transmitted even by experimental inoculation, and the slowness with which the disease develops, even in the severe lepromatous case. These two factors might mean that a chemotherapeutic agent which affected the vitality of the organisms only slightly might still possibly just turn the balance and enable the body to combat and overcome the infection in the long period of time available.

\section{Sulphone Therapy in Practice.}

When sulphones were first studied and were found to be active against the tubercle bacillus not only in vitro but, to some extent, in the guinea pig, the chances of chemotherapy of leprosy seemed better; but when sulphones were found to exert little influence on tuberculosis in man, the chances again seemed to recede.

But there were those in the United States who were not prevented by the theoretical difficulties outlined above from making the trial of these drugs in leprosy, a long and difficult trial; gradually it appeared that good results were being seen, and as more work is being done the reported results appear to be improving. Although the treatment has now been used for several years in a few centres, the initial wave of optimism has not yet been replaced by a trough of depression; nor do we think that this is likely as long as scientific caution is used in reporting results of treatment.

These remarks must not be read as indicating that the problem of leprosy treatment has been solved. The sulphone treatment has grave limitations; it is dear; it has to be given for periods varying perhaps from two years upwards, and its ultimate results are not yet clear. Its value in the non-lepromatous forms of the clisease has not been demonstrated; in fact it is reported to be of no value in such cases. The writer, however, having had over twenty years' experience of leprosy and its treatment with other remedies, and having now had some experience of sulphone treatment, feels that a very definite advance has been made. His practical and personal experience of sulphone treatment is confined to the last eight months and covers less than 200 cases, 
mainly lepromatous. The results are obtained in the more severe infectious progressive lepromatous forms of the disease, which have been least amenable to other forms of treatment, and which present the great problem in treatment. Further, the treatment can be given orally and in ambulant patients and, contrary to some reports, it is found to be well tolerated, toxic effects being few and usually not serious. The beneficial effects are seen in varying degree in almost every lepromatous case treated for a period of six months or more, the improvement being both clinical and bacteriological.

\section{Mode of ACtion of Sulphones.}

The question arises, how do the sulphones act in leprosy? The sulphones were selected for use in leprosy because of their action on acid-fast bacilli in vitro and in experimental animals; surely it is unnecessary to advance quite a different explanation of therr activity in leprosy in man. There seems no adequate reason to doubt that the action in leprosy is on the bacilli; the dimunition in the number of bacilli and the change in morphology of the bacilli scen in cases under prolonged sulphone treatment can be very striking. It is, however, not clear whether the action is bacteriocidal or merely bacteriostatic.

But it may be asked "If the action is a clirect one on the bacilli, how can the bacilli (or at any rate acid-fast material in more or less bacillary form) persist for years during sulphone treatment, even if in steadily diminishing numbers?"

To this question there appear to be two possible answers. Few workers who have not made a special study of the matter realise the fantastically large numbers of bacilli present in leprous lesions in a lepromatous case, and the extraordinary way in which even dead lepra bacilli can persist in the body in acid-fast bacillar; form. The enormous numbers of bacilli in lesions is shown by a microscopic study of sections of leprous nodules properly staine? to show bacilli, and also from the study of bacillary counts in emulsions made by grinding up leprous nodules of known size. Hanks (Ref. I8) has shown that nodules may frequently contain more than an American billion (one thousand millions) of bacilli per cubic centimetre. The long persistence of even dead bacilli in living tissue is shown by a study of animals injected with killed lepra bacilli, or by making smears from the skin at the site of intradermal injections of lepromin in a healthy person who has been lepromin tested, i.e., who has had injected into the dermis about one million dead bacilli; bacilli may be recovered over periods up to one year and even more. 
A consideration of these facts shows that there is nothing surprising about the long persistence of acid-fast bacilli in lepromatous cases treated with sulphones, bacilli persisting long after clinical activity of the disease has subsided. It is not impossible that these persisting bacilli are dead, although this cannot be proved.

For the discharge of patients we still have to maintain the criterion of the failure to demonstrate acid-fast bacilli in the tissues examined by the usual methods, but it should be realised that this criterion may be a very harsh one in certain cases. It would be interesting to study patients who, after say two or three years sulphone treatment, have ceased treatment whilst still showing bacilli in the tissues, and to see whether these bacilli slowly disappear in spite of the cessation of treatment.

The second answer to the query regarding the persistence of acid-fast bacilli in sulphone treated cases is concerned with the possibility that the bacilli may become sulphone resistant and thus be enabled to survive and persist though in diminished numbers. If this were the true explanation, a high incidence of relapse would be expected in patients discontinuing sulphone treatment. No one has yet reported such a finding, perhaps because too few patients have, as yet, been treated and then observed for long enough. But the evidence so far available is that, after sulphone treatment, relapse, though not unknown, is not common.

At this point of our consideration of possible modes of action of sulphones, a little speculation might be useful.

Suppose we had a chemotherapeutic agent so efficient that it would kill off all the bacilli in a lepromatous case in a few days or weeks; what changes could reasonably be expected to follow such treatment in a patient with lepromatous leprosy? Firstly, the dead bacilli would almost certainly persist for many months, if not for years; clinical activity due to the multiplication and dissemination of bacilli in the body would cease rapidly, but symptoms caused merely by the presence of bacilli or by the reaction of the body to the bacillary products liberated by the slow disintegration of the dead bacilli would probably disappear much more slowly. Secondly, we should expect to find no sudden change in the histopathology of the lesions; the active leprous changes would probably slowly be replaced by atrophy and fibrosis, as the dead bacilli slowly disintegrated. Thirdly, we should not expect a rapid change in immunology, the lepromin test giving positive results in cases previously negative, The 
results of this test normally show only minor variations in degree and not in kind, with variations in clinical and bacteriological activity in any given case.

In other words, the rapid destruction of all the bacilli in the body by our imagined chemotherapeutic agent of high potency would probably be followed by the same sequence of events, but occurring with greater rapidity, as we now see with sulphone therapy.

The point of this specculation is this; that in the slow subsidence of clinical activity of leprosy under sulphone therapy, in the slow appearance of the acid-fast bacilli from the lesions, there is nothing inconsistent with the view that the action of the sulphones is a direct one on the bacilli.

This is the view of Faget et al., (Ref: 5) who, summarising five years observation of promin therapy, state " the action of promin appeared to be a chemotherapeutic effect on the aetiological agent of leprosy." In the same article, however, they make the following remark, "the important finding is that promin appears to eliminate bacillary infection of the blood vessels and blood stream, thereby preventing the formation of new lesions." This is probably true, but it would appear not to be the whole truth. It cannot explain the subsidence of the lesions present before treatment started. It appears probable that sulphone is taken up by tissue cells, particularly reticulo-endothelium, including the actual cells of the leprous lesions, and that it exercises an influence on the bacilli in the lesions, which are mostly intracellular.

It is worthy of note that the therapeutic effect of sulphones ii leprosy can be produced by a dosage and with blood levels which, on theoretical grounds and on evidence gained by studies of tuberculosis in guinea pigs, would appear to be hopelessly inadequate; but this is perhaps another matter in which practical experience is of far more value than theory. There is some evidence that sulphones are concentrated in the leprous lesions.

\section{Factors Influencing The Evaluation of Sulphone Therapy.}

The enthusiasm, or lack of it, with which individual workers have greeted sulphone treatment has varied, and has obviously been influenced by a number of factors. Thus the value attached to sulphone treatment by any physician tends to be influenced by the severity of the leprosy with which he deals (for leprosy varies widely in severity. in different countries and peoples), and upon the value he attaches to other forms of treatment, particularly hydnocarpus treatment. For example, in the Leprosarium at 
Carville, Louisiana, where sulphone trcatment originated, the cases of leprosy are mostly of the severe lepromatous type and hydnocarpus treatment has never given satisfactory results; therefore sulphone treatment is regarded by patients, even more than by physicians, as a great triumph, although Faget and others (Ref. 5) working there have not hesitated to state that "Promin is the best treatment of leprosy ever used there." From India, on the other hand, with leprosy on the whole less severe than in some countries, publications on sulphone treatment have been few and rather lukewarm (Refs: I5, I6, I7). It is, therefore, striking to find among those who write most enthusiastically of sulphone treatment Muir (Refs: IO, II and I2) who was for many years perhaps the strongest advocate of hydnocarpus treatment. Muir (Ref: I2) states frankly of diasone, "During more than twenty-seven years of experience of leprosy treatment and the trial of many drugs, the author has not seen any remedy for definite lepromatous cases of leprosy which has given such uniformly favourable results ", a striking statement from such a worker, yet one which the present writer, even from his more limited experience of sulphone treatment would support. Reports of other workers from other countries are, so far, few in number, but practically every one is favourable.

A worker's attitude to sulphone treatment will almost certainly be influenced by his views on the evolution of leprosy. For example, a worker who believes that tuberculoid cases commonly become lepromatous, that hydnocarpus treatment can prevent this change, and that sulphones are of no value in tuberculoid cases, will be likely to consider sulphone treatment to be of limited value. The present writer holds none of these beliefs, and tends to think that the value of sulphones in tuberculoid cases has not yet been thoroughly studied with due consideration of what might reasonably be expected from chemotherapy in such cases.

There is no obvious reason why sulphones (if their action is on the bacilli, as it appears to be), should not be of value in the treatment of all active cases of leprosy irrespective of type. All leprous lesions are caused by bacilli; either by the presence of large numbers of bacilli with little tissue reaction, e.g., in the lepromatous type, or by the reaction, probably allergic in nature, of the tissues to the products of the relatively small number of bacilli, possibly liberated by the destruction of some of them, e.g., in the tuberculoid type. So far, only a few tuberculoid cases have been treated, mostly for limited periods, and results do not appear to be 
entirely negative, although the evaluation of any treatment in tuberculoid cases is notoriously difficult because of the marked tendency to spontaneous subsidence.

Without wishing to discuss in any detail the relative merits of sulphone and hydnocarpus treatment, one may mention a few points. The main difference between results of hydnocarpus treatment and sulphone treatment appears to be in the proportion of cases responding. Hydnocarpus treatment can, on occasions, produce marked and progressive improvement in lepromatous cases, probably as impressive as that produced by sulphones, but the proportion of treated cases showing satisfactory improvement is much lower. Cochrane, a strong advocate of hydnocarpus treatment has recently stated (Ref: I7) that "from 30\% to $50 \%$ of early cases of lepromatous leprosy respond satisfactorily to properly administered hydnocarpus therapy." How can this possibly compare with the response to sulphones seen in almost every lepromatous case treated, whether early or advanced? Muir (Ref: II) has recently emphasised the constancy of the response to sulphones and others have reported similarly. No more need be said on this point. Cochrane (Ref: I7) also states that the " sulphones are the treatment of choice in advanced lepromatous cases "; this statement might be read (one feels incorrectly) as indicating that he thinks sulphones are less effective in early cases. Faget et al., (Ref: 5) have reported that the best results of sulphone treatment are seen in the early lepromatous cases. Cochrane (Ref: I7) also points out with ample justification that the high cost of sulphones at present makes quite impossible their wide adoption in poor countries, such as India. The same may be said of most of Asia and Africa. This factor is, however, less potent in other countries than in India where, presumably, hydnocarpus oil is obtainable in good quality at a reasonable price. Other countries, such as West Africa, are obtaining from India hydnocarpus oil, often of miserably poor quality, and at no cheap price, and trouble with injections is very common and serious.

Undoubtedly, hydnocarpus treatment will continue to be used for some time yet, but where sulphones are available they should be the treatment of choice in lepromatous cases, if not in others. Some workers will undoubtedly prefer to treat patients with both remedies simultaneously; others will welcome the opportunity of discarding the painful and tedious injection treatment, particularly the intradermal injections which efficient hydnocarpus treatment nccessitates. 
The Significancl: OF SUlphONI: Thi:RAPy,

To the writer's mind a very important fact is this; that for the first time a group of chemotherapeutic agents, not seriously toxic in therapeutic doses, and capable of being given, some of them continuously, for many months on end, has been found to exert a very definite influence upon the bacterial disease, leprosy, which possibly of all bacterial diseases would, on general grounds, be expected to be the least likely to be susceptible to such treatment. The scope of chemotherapy has been extended in quite a new and, to many of us, an unexpected direction. This fact is important, not only to leprosy work, but to other fields of medicine.

One cannot help feeling some satisfaction that leprosy treatment, which has for so long made such slow progress, has now made a definite advance, and further one hopes that other fields of therapeutics may benefit from this advance. Our experience of sulphones in leprosy may help workers studying other chronic infections, particularly acid-fast bacillary infections.

This new development, the sulphone treatment of leprosy, should give very welcome encouragement and stimulus to antileprosy work and to individual workers everywhere.

The influence of sulphone treatment on anti-leprosy work is only just beginning to be felt. At the National Leprosarium, Carville, Lousiana, where the treatment started in I94I, the change for the better in the outlook is apparent from the publications of medical staff and also of the patients in the journal which they publish, and from the increase in the discharge rate; one is informed that this optimistic outlook is very apparent to visitors. A similar change will doubtless be seen elsewhere as patients realise that the chances of arresting the lepromatous form of the disease are greatly improved.

How this will affect anti-leprosy work remains to be seen. One may expect increased demands for sulphone treatment and for admission to institutions using it.. An increased discharge rate of healed lepromatous cases may be expected, which may necessitate increased staff and facilities for supervision and reexamination of discharged cases. There may be the need for the modification of the types of leprosy institutions and methods of work, since more patients should be effectively treated and discharged, and fewer patients will remain indefinitely, and finally die in leprosy institutions.

Cochrane (Ref: I6) has written rather critically of sulphone therapy, stressing that Indian patients do not tolerate it well, and 
also that it may have a harmful effect on prevention by isolation. which he considers more important than treatment. He is almost alone in finding the treatment badly tolerated. One can agree that in the prevention of leprosy the establishment and maintenance of a reasonable degree of isolation of lepromatous cases, particularly from children and young people, is of paramount importance; but the present writer would go further and state his opinion that without a treatment much more effective in lepromatous cases than those used in the past, attempts at isolation of sufficient lepromatous cases of leprosy to influence the spread of leprosy in the general population are, in most countries, greatly handicapped, if not doomed to failure.

To put the matter briefly; usually a person with leprosy will undergo and maintain isolation only if it is accompanied by a treatment sufficiently effective to give him a good chance of the disease being arrested, and the isolation therefore ended, within a reasonable period, a few years at the most. In most countries where leprosy is common, without this inducement of an effective treatment, so many patients will refuse isolation if voluntary, or avoid isolation if compulsory, that the control of leprosy will be impossible. In these circumstances any improvement in treatment is potentially an aid to prevention. It remains to be seen whether sulphone treatment is, or can be made, sufficiently effective to afford this aid to preventive work which is so much needed.

It is true that there are dangers to be guarded against; improved treatment must not be regarded as removing the need for a reasonable degree of isolation; the treatment must be given efficiently and must not cease as soon as clinical activity of the disease disappears, for this might only enable patients to live longer and spread the infection more. The methods to be adopted to avoid these dangers will vary in different countries, but it should not be impossible to devise suitable methods.

To conclude on this point: the writer feels that sulphone treatment wisely used should not hinder preventive work but should aid it.

Finally, there is the effect of this new development on the individual leprosy worker. While knowledge and other aspects of leprosy has steadily increased, until now no fundamental change in treatment has occurred in the last forty years or so, since injection treatment with hydnocarpus oil and its preparations became established. Improvements have been made in the preparations used and in methods of administering them, but the basic idea of the treatment is still the same, and the limitations 
of the treatment have long. been apparent. Now a definite new development which is full of promise has appeared, and a new field of investigation has been opened up. It is to be hoped that more medical men will be attracted to take up leprosy work, to exploit these new advances to the full, and make further advances.

There is now for the leprosy worker a feeling that gives some satisfaction, that with the sulphones he is using treatment that has a rational basis, even if little is known of the mode of the action. He can feel that here rational and planned research in chemistry, in bacteriology, in experimental animals and finally in human beings has given results even in the disease, leprosy. which often appears so irrational in its behaviour.

We must remember, however, that theorising, however rational, must never replace practical experiment; if it had been allowed to do so, the sulphones might never have been tried in leprosy, because the theoretical difficulties. seemed so great.

\section{REFERENCES.}

- 1. Faget, G. H. et al. (1943) Pub. Health Rep. 58, 1729.

- 2. Faget, G. H. and Pogge, R. C. (1945) Neu Orleans, Med. E Surg. Jl. 98,145 .

. 3. Faget, G. H. (1935) Pub. Health Rep., 60, 1165.

- 4. Faget, G. H. et al. (1946) Pub. Health Rep. 61, 960).

. 5. Faget, G. H. ct al. (1946) Int. Jl. of Lep., 14, 30.

- 6. Faget, G. H. (1947) Int. Jl. of Le'p., 15, 1.

- 7. Fernandez, J. M. M. and Carboni, E. A. (1936) Int. Jl. of Lep., 14, 19.

- 8. Fite, G. L. and Gemar, F., (1946) Southern Med. Jl. 39, 277.

- 9. Mom, Arturu, M. (1946) Prensa Med. Arg. 48, 2390.

10. Muir, E. (1944) Int. Jl. of Lep., 12, 1.

. 11. Muir, E. (1946) Lep. Ret., 17, 87.

. 12. Muir, E. (1947) Brit. Med. Jl. (7.6.47) 799.

- 13. Wharton, L. H. (1946) Lep. Re". 17, 96.

. 14. Davey, T. F. (1948) Lep. Rev. 19, 55.

- 15. Dharmendra (1948) Let). in India 20, 71.

16. Cochrane, R. G. (1947) Brit. Med. Jl. (19.7.47) 110.

17. Cochrane, R. G. (1948) "Star", Carville, Louisiana, 7, 10.

- 18. Hanks, J. H. (1945) Int. Jl. Lep. 13, 25. 